\title{
Conformational changes of fibrinogen in dispersed carbon nanotubes
}

\author{
This article was published in the following Dove Press journal: \\ International Journal of Nanomedicine \\ 3 August 2012 \\ Number of times this article has been viewed
}

\author{
Sung Jean Park' \\ Dongwoo Khang ${ }^{2}$ \\ 'College of Pharmacy, Gachon \\ University, Yeonsu-gu, Incheon, \\ South Korea; ${ }^{2} \mathrm{School}$ of Nano \\ and Advanced Materials Science \\ Engineering and Center for PRC \\ and RIGET, Gyeongsang National \\ University, Jinju, South Korea
}

Correspondence: Dongwoo Khang School of Nano and Advanced Materials Science Engineering, Gyeongsang National University, Jinju, 660-70I, South Korea Tel +82557630217

Fax +82557630217

Email dkhang@gnu.ac.kr

\begin{abstract}
The conformational changes of plasma protein structures in response to carbon nanotubes are critical for determining the nanotoxicity and blood coagulation effects of carbon nanotubes. In this study, we identified that the functional intensity of carboxyl groups on carbon nanotubes, which correspond to the water dispersity or hydrophilicity of carbon nanotubes, can induce conformational changes in the fibrinogen domains. Also, elevation of carbon nanotube density can alter the secondary structures (ie, helices and beta sheets) of fibrinogen. Furthermore, fibrinogen that had been in contact with the nanoparticle material demonstrated a different pattern of heat denaturation compared with free fibrinogen as a result of a variation in hydrophilicity and concentration of carbon nanotubes. Considering the importance of interactions between carbon nanotubes and plasma proteins in the drug delivery system, this study elucidated the correlation between nanoscale physiochemical material properties of carbon nanotubes and associated structural changes in fibrinogen.
\end{abstract}

Keywords: carbon nanotubes, fibrinogen, nanotoxicity, conformational change, denaturation

\section{Introduction}

Understanding the fundamental interactions of plasma proteins with nanoparticles such as carbon nanotubes (CNTs) and gold nanoparticles is critical in the evaluation of the inflammatory and blood coagulation process of these materials for potential drug-delivery applications. ${ }^{1-3}$ The use of nanoparticles as carriers for biomolecules has been widely studied, ${ }^{4}$ the bioavailability of nanoparticles dependent on the cellular uptake and effective biodistribution of nanoparticles throughout the body for controlled drug delivery. ${ }^{5,6}$

It has been widely accepted that nanometer-scale surface topography and chemistry greatly contribute to the protein adsorption on nanoparticles. For example, recent studies have emphasized the importance of the conformational state of an adhered protein, as the activity of a protein is contingent on its structural conformation. ${ }^{7}$ In accordance with this point of view, it was hypothesized that the interaction between nanoparticles and plasma proteins can determine the cytotoxicity of nanoparticles. ${ }^{8,9}$ The assembly of proteins surrounding nanoparticles is governed by hydrophobic and/or electrostatic interactions at the surface of the nanoparticles. ${ }^{10,11}$ Although the protein corona forms soon after the exposure of the surface of nanoparticles to blood plasma, ${ }^{12}$ the intrinsic surface properties of nanoparticles instantly induce conformational changes in blood proteins, and in turn modulate nanoparticle toxicity on cells. ${ }^{3}$

Fibrinogen, a well-known regulator of the host inflammatory response, triggers hemostasis and leukocyte activation through stimulation of the integrin receptor, Mac-1. ${ }^{13}$ 
While the molecular mechanism behind leukocyte activation remains unclear and controversial, the structural state of fibrinogen is believed to play an important role in high affinity binding with Mac-1. Notably, immobilized fibrinogen acts as a better ligand than soluble fibrinogen for Mac-1, suggesting that the conformational state of fibrinogen is critical to leukocyte activation. In this regard, the effect of nanoparticles on the conformational change of fibrinogen may provide a molecular level understanding of the inflammatory response to nanoparticles. Indeed, studies have also shown that enhancing the density of gold nanoparticles induced macrophage activation via the Mac- 1 receptor. ${ }^{2}$

Fibrinogen is a rod-shaped protein with three chains ( $\mathrm{A} \alpha, \mathrm{B} \beta$, and $\gamma$ ) that are linked together by 29 disulfide bonds ${ }^{14}$ to form a $45 \mathrm{~nm}$-long dimer protein $(\mathrm{A} \alpha \mathrm{B} \beta \gamma)_{2}$, with monomers bound to each other via disulfide bridges. ${ }^{15}$ All three chains are linked together at each end of the $\alpha$-helical coiled coils. ${ }^{16}$ The protein has globular domains at each end and is connected in the middle by $\alpha$-helical coiled-coil rods. Thus, the secondary structure of fibrinogen possesses a helical component. Spectroscopic analysis can provide general information regarding the conformational changes of $\beta$ sheets, $\alpha$ helices and coils (including turn structures) on nanoparticles. ${ }^{7}$ For example, systematic changes on the surface curvature of silica nanoparticles alters the secondary structures of fibrinogen on hydrophilic and hydrophobic surfaces. $^{17}$

The theoretical and experimental interactions of CNTs with blood plasma proteins have been investigated in previous studies. For example, molecular dynamics on the conformational changes of albumin in response to CNTs has previously been discussed. ${ }^{18}$ In addition, it was demonstrated that the conformational changes were associated with changes in $\alpha$ helix peptides on single walled CNTs (swCNTs) ${ }^{19}$ and the transformation from an $\alpha$ helix to a $\beta$ sheet on graphene ${ }^{20}$ using classical molecular dynamics. The chemical functional groups on multiwall CNTs were also analyzed based on their associated fluorescence and circular dichroism (CD). For example, CD studies on the conformational changes in terms of CNT concentration on albumin were also examined. ${ }^{21}$

However, to date there have been no studies addressing or comparing the roles of water dispersion and the concentration of swCNTs on the conformational change of fibrinogen, including thermal instability. The instability of the fibrinogen structure may be changed by enhancing the interaction sites between protein residues and swCNTs can enhance the probability of conformational change. Protein denaturation and thermal stability (or melting temperature) of fibrinogen by enhancing orders of carboxylate and increasing concentrations of swCNTs were analyzed to support the evidence of conformational change of secondary and tertiary structures (by CD) in fibrinogen.

\section{Materials and methods Controlling carboxylation order of swCNTs}

Purified swCNTs were purchased (900-1351; SES, Houston, TX) and oxidized by different methods to generate different orders of carboxylate CNTs. To generate less carboxyl groups (COOH-1), $10 \mathrm{mg}$ of swCNTs were mixed with $3 \mathrm{~mL}$ of $\mathrm{H}_{2} \mathrm{SO}_{4}$ and $1 \mathrm{~mL}$ of $\mathrm{HNO}_{3}$. The mixed solution was stirred with a magnetic stir bar for 14 hours at room temperature. Next, the solution was diluted with deionized water (1:200 v/v), filtered (200 nm pore size PTFE; Millipore, Billerica, MA), and washed out several times to completely remove any residual solvent on the CNTs. swCNTs were then dried in a vacuum oven at $60^{\circ} \mathrm{C}$ overnight. To introduce more carboxyl groups (COOH-2), $10 \mathrm{mg}$ of swCNTs were mixed with $3 \mathrm{~mL}$ of $\mathrm{H}_{2} \mathrm{SO}_{4}$ and $1 \mathrm{~mL}$ of $\mathrm{HNO}_{3}$. The prepared solution was stirred using a magnetic stir bar for 30 minutes at room temperature and homogenized in a water sonicator for 99 minutes. The solution was then diluted, filtered and dried as previously described. To generate the greatest order of carboxyl groups (COOH-3), $20 \mathrm{mg}$ of swCNTs was immersed in $9 \mathrm{~mL}$ of $\mathrm{H}_{2} \mathrm{SO}_{4}$ and $3 \mathrm{~mL}$ of $\mathrm{HNO}_{3}$ by sonication in a water bath for 30 minutes, and stirred with a magnetic bar for 24 hours at $50^{\circ} \mathrm{C}$. The samples were washed, filtered, and dried following the same protocols as described previously.

\section{Size and surface chemistry of carboxylate swCNTs}

The evidence of carboxyl groups presented on the CNT surface was visualized with a high-resolution field emission transmission electron microscopy (FE-TEM; JEM 2100F; JEOL, Tokyo, Japan) operating at $200 \mathrm{kV}$ with a slow scan CCD camera (Gatan, Pleasanton, CA). All swCNT samples were diluted in ethanol and sonicated for 2 minutes before undergoing field emission transmission electron microscopy procedures. Fourier transform infrared spectroscopy (Nicolet Avatar 320; Nicolet, Pasadena, CA) was performed to analyze carboxyl formation on swCNTs. The samples were prepared by grinding the CNTs in an agate mortar with $\mathrm{KBr}$ and subjected to $70 \mathrm{MPa}$ of applied pressure. The transparent flakes were analyzed within the mid infrared 
wavelengths (400 4000 $\mathrm{cm}^{-1}$ ). To analyze the oxide amount on each carboxylate swCNTs, X-ray photoelectron spectroscopy (Thermo Fisher Scientific, Rockford, IL) was performed to determine the atomic weight ratio. $\mathrm{K}_{\alpha}$ radiation was used from an $\mathrm{Al}$ anode with a beam size of $400 \mu \mathrm{m}$. The energy step size was $0.1 \mathrm{eV}$ with ten scans. Electron spectroscopy for chemical analysis was carried out for C1s (carbon) and O1s (oxygen) peaks to compare comparative areas of each curve representing the atomic weight ratio. Particle size analysis (laser light-scattering method, ELS-6000; Otsuka, Hirakata-shi, Japan) was performed to analyze particle size and the diffusion coefficient of used swCNTs. Quantitative analysis of the weight amount of functionalized groups (carboxyl) was analyzed by thermal gravimetric analysis (Q50; TA Instruments, New Castle, DE). Ten micrograms of swCNTs were heat-treated at $60^{\circ} \mathrm{C}$ for 3 hours in a vacuum prior to thermal gravimetric analysis to evaporate remnant water molecules from the surfaces. Thermal gravimetric analysis (TGA: Q50, TA Instrument, New Castle, DE, USA) was performed with increasing temperature at $10^{\circ} \mathrm{C} /$ minute with a $\mathrm{N}_{2}$ supplement of $100 \mathrm{~mL} /$ minute.

\section{Fibrinogen adsorption assays}

Fibrinogen (1 mg/mL, F4883; Sigma, St Louis, MO) was gently mixed with various orders of carboxylate swCNTs (ie, COOH-1, -2 and -3 at a density of $50 \mu \mathrm{g} / \mathrm{mL}$ ) and incubated for 4 hours under standard cell culture conditions. In addition, swCNTs samples featuring a fixed carboxylate order (COOH-3) were prepared at varying densities (ie, 26, 64 and $128 \mu \mathrm{g} / \mathrm{mL})$, mixed with fibrinogen $(1 \mathrm{mg} / \mathrm{mL})$, and incubated for 4 hours. After incubation, each solution was centrifuged at $12000 \mathrm{rpm}$ for 30 minutes to completely remove the suspended swCNTs from the fibrinogen solution. The remaining fibrinogen found in the supernatant corresponded to fibrinogen that did not interact with the swCNTs. The concentration of attached fibrinogen was measured using a bicinchoninic acid protein assay kit (Coomassie Plus 23236; Thermo Fisher Scientific) and measured using a spectrophotometer (Asys UVM 340; Biochrom, Berlin, Germany) at an excitation wavelength of $595 \mathrm{~nm}$. The amount of fibrinogen in solution was quantified by extrapolation from the resulting linear standard curve $\left(\mathrm{r}^{2}>0.997\right)$.

\section{CD spectroscopy and temperature scan}

To characterize the secondary structure of fibrinogen in the presence of CNTs, a CD analysis was performed using a $2 \mathrm{~mm}$ path length cuvette in a Chirascan CD spectrometer (Applied Photophysics, London, UK) at $20^{\circ} \mathrm{C}$. CD measurements were carried out in the wavelength range between $195 \mathrm{~nm}$ and $260 \mathrm{~nm}$. The purchased human fibrinogen (F4883; Sigma) was dissolved in phosphate-buffered saline ( $\mathrm{pH} 7.4$ ) and mixed with $\mathrm{COOH}-1,-2$, and -3 swCNTs. The final concentration of fibrinogen used in the $\mathrm{CD}$ measurements was $750 \mu \mathrm{g} / \mathrm{mL}$ and the final concentration of COOH-1, 2, and 3 was set to $100 \mu \mathrm{g} / \mathrm{mL}$. Mixtures of fibrinogen containing nanoparticles were pre-incubated in phosphate-buffered saline (pH 7.2; Gibco, Grand Island, NY) for 30 minutes at $37^{\circ} \mathrm{C}$. The resultant spectra were corrected based on buffer and nanoparticle controls. Both the buffer and the nanoparticles did not show any significant CD signals within the given range of wavelengths (data not shown). Thermally induced denaturation of secondary structures was monitored by measuring the signal change at $220 \mathrm{~nm}$. Temperature scanning was conducted at a rate of $1^{\circ} \mathrm{C} /$ minute across the temperature range: $20^{\circ} \mathrm{C}$ to $80^{\circ} \mathrm{C}$. To monitor the effect of various concentrations of $\mathrm{COOH}-3$ swCNT, the $\mathrm{CD}$ measurements were performed for wavelengths between $195 \mathrm{~nm}$ and $260 \mathrm{~nm}$. The systematic controls of $\mathrm{COOH}-3$ swCNT concentrations in the fibrinogen samples were set to $26 \mu \mathrm{g} / \mathrm{mL}, 64 \mu \mathrm{g} / \mathrm{mL}$, and $128 \mu \mathrm{g} / \mathrm{mL}$ respectively. The $\mathrm{COOH} 3$-swCNTs and fibrinogen $(750 \mu \mathrm{g} / \mathrm{mL})$ were preincubated for 30 minutes at $37^{\circ} \mathrm{C}$ prior to measurement. The change of secondary structures in the fibrinogen samples was analyzed with the corresponding CD analysis software, CDNN (CD spectra Deconvolution Ver. 2.1; Gerald Böhm, Bioinformatics, University of Halle, Germany).

\section{Results and discussion Controlling carboxylation order of swCNTs}

Transmission electron microscopy images verified the generation of surface carboxyl groups on swCNTs (Figure 1A). Fourier transform-infrared signals showed significant absorption peaks corresponding to covalent $\mathrm{C}=\mathrm{O}$ bonds at 1230 and $1720\left(\mathrm{~cm}^{-1}\right)$, whereas unmodified swCNTs did not exhibit any notable peaks around the $\mathrm{C}=\mathrm{O}$ energy (Figure 1B). Increasing the order of carboxyl functional groups inhibited the aggregation of individual swCNTs and enhanced the dispersion of CNT in water and in cell culture media (Figure 1C). At higher dispersion (COOH-3), no deposition was observed as densities were increased from 24 to $128 \mu \mathrm{g} / \mathrm{mL}$ (Figure 1D). Figure 1E depicts contacted fibrinogen on swCNT.

The XPS results showed increasing intensities at $290 \mathrm{eV}$ (O1s: oxide) as the carboxyl amount increased from $13.5 \%$ to $18 \%$ (surface atomic \%) (Figure $2 \mathrm{~A}$ and $\mathrm{B}$ ). The average length of each type of swCNTs was $230 \mathrm{~nm}, 190 \mathrm{~nm}$ 

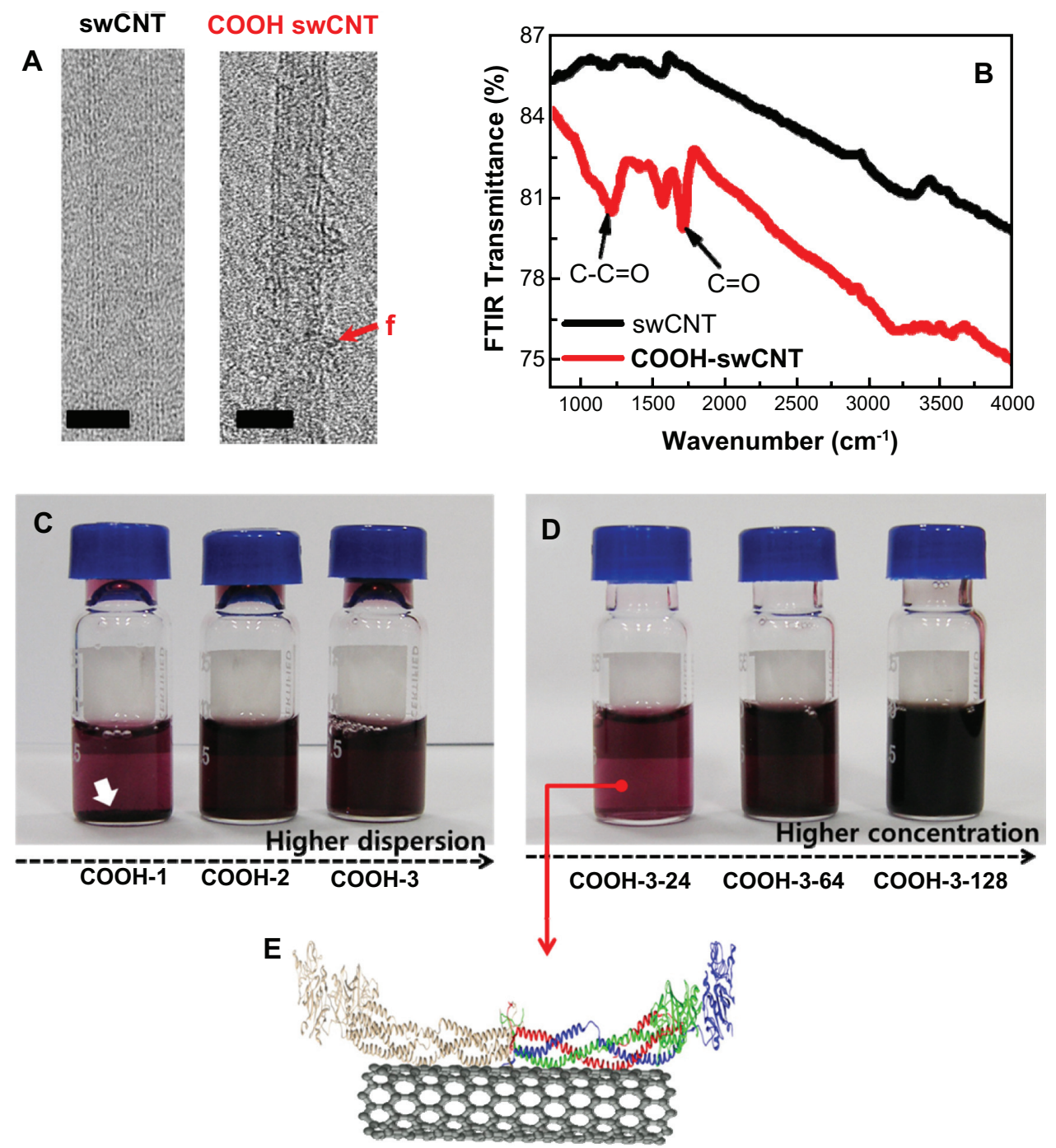

Figure I Physiochemical properties, dispersion and concentration of carboxylate swCNTs. (A) TEM images show pristine swCNTs and carboxylate (COOH) swCNTs. (B) FTIR absorbance shows significant carboxylate formation at 1230 and $1720\left(\mathrm{~cm}^{-1}\right)$ on $\mathrm{COOH}$-swCNTs. (C) Higher dispersion shows highly dispersed state in media without sediment. (D) Various $\mathrm{COOH}-3$ swCNT concentration ranges from 24 to $128 \mu \mathrm{g} / \mathrm{mL}$ in media. (E) A cartoon of the 3D structure of fibrinogen dimers adsorbed on swCNTs.

Notes: The red f shows functionalized carboxyl groups. Bars in TEM show $2 \mathrm{~nm}$. White arrows designate sediment of swCNTs in DMEM due to less carboxylate swCNTs $(\mathrm{COOH}-\mathrm{I})$ compared with $\mathrm{COOH}-2$ and $\mathrm{COOH}-3$ swCNTs. Red, green, and blue colors represent the A $\alpha$ chain, $\mathrm{B} \beta$ chain, and $\gamma$ chain, respectively. The structure of human fibrinogen (PDB code: 3GHG) was obtained from the PDB database (http://www.rcsb.org).

Abbreviations: PDB, Protein Data Bank; TEM, transmission electron microscopy.

and $145 \mathrm{~nm}$ for $\mathrm{COOH}-1, \mathrm{COOH}-2$ and $\mathrm{COOH}-3$, respectively (Figure 2C). Due to enhanced hydrophilicity from the increased surface oxide, the $\mathrm{COOH}-3$ samples demonstrated a $50 \%$ greater diffusion constant $\left(\mathrm{cm}^{2} / \mathrm{sec}\right)$ than that of COOH-1 (Figure 2D). The carboxyl weight percentages of $\mathrm{COOH}-1,2$, and 3 were more than $12 \%, 21 \%$ and $22 \%$ (at $600^{\circ} \mathrm{C}$ ), respectively (Figure $2 \mathrm{E}$ and $\mathrm{F}$ ).

As the average length of individual CNT differed with the order of carboxyl groups (Figure 2B-D), it was hypothesized that a larger carboxyl-covered surface area was exposed to fibrinogen in $\mathrm{COOH}-3$ than in the $\mathrm{COOH}-1$ samples for a given volume. Although the increased order of carboxyl groups altered both the surface chemistry and physical size of the swCNT samples, the effects of size may have played a larger role in protein interactions as the chemical interaction between fibrinogen and surface oxide is nonspecific and noncovalent. The $\mathrm{COOH}-3$ swCNTs provided a more hydrophilic environment (ie, more surface negative charges) for the interactions with fibrinogen than the $\mathrm{COOH}-1$ swCNTs.

\section{Conformational changes of fibrinogen with increasing dispersion of swCNTs}

Conformational changes of fibrinogen in terms of dispersion order (ie, hydrophilicity) were observed by $\mathrm{CD}$ spectrometry. The CD spectrum of fibrinogen indicated that fibrinogen undergoes notable structural changes depending on the order 

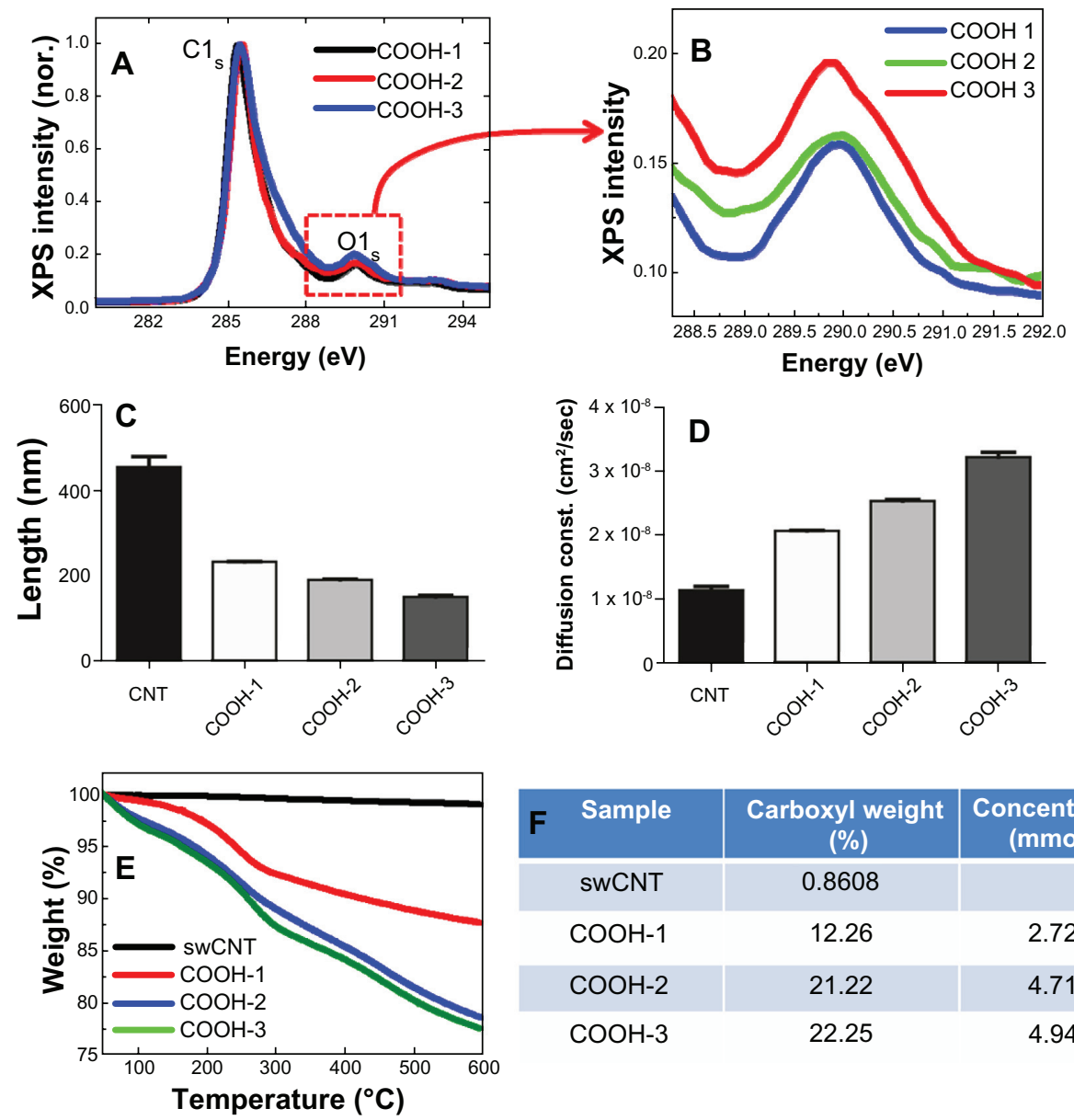

\begin{tabular}{|c|c|c|}
\hline Fample & $\begin{array}{c}\text { Carboxyl weight } \\
(\%)\end{array}$ & $\begin{array}{c}\text { Concentration } \\
(\mathrm{mmol} / \mathrm{g})\end{array}$ \\
\hline swCNT & 0.8608 & \\
\hline COOH-1 & 12.26 & 2.724 \\
\hline COOH-2 & 21.22 & 4.716 \\
\hline COOH-3 & 22.25 & 4.944 \\
\hline
\end{tabular}

Figure 2 Controlling carboxyl order on swCNTs and associated physiochemical materials properties. (A) XPS shows surface oxide (carboxyl groups) on swCNTs. (B) Magnified XPS intensity at oxygen (OIs) shows increasing order of surface oxides as the carboxyl groups are increased on swCNTs. (C) Length of pristine swCNTs shows above $400 \mathrm{~nm}$ on average. As carboxyls increase, the length of carboxyl swCNTs decreases from 200 to I $40 \mathrm{~nm}$. (D) Diffusion constant in distilled water (DI) shows enhanced hydrophilicity as the carboxyl groups are increased on swCNTs. (E and F) TGA mass curve shows carboxyl concentration from I $2 \%$ to $22 \%$ by atomic weight $\%$ to $600^{\circ} \mathrm{C}$.

Abbreviations: XPS, X-ray photoelectron spectroscopy; TGA, thermal gravimetric analysis.

of the carboxyl functional groups (Figure $3 \mathrm{~A}$ and $\mathrm{B}$ ). The spectrum of free fibrinogen showed two negative minima at $208 \mathrm{~nm}$ and $222 \mathrm{~nm}$ respectively, which are indicative of an $\alpha$ helical structure (Figure 3A). The addition of swCNTs of increasing carboxyl order corresponded to increased intensity (absolute value) at $208 \mathrm{~nm}$ and $222 \mathrm{~nm}$ without the shift of the negative minima, suggesting that the fibrinogen underwent a structural change (Figure 3A). The role of swCNTs dispersion on fibrinogen structure was significant when $\mathrm{COOH}-2$ and -3 swCNT were compared with $\mathrm{COOH}-1$ swCNT. The ratio of $\mathrm{CD}$ intensities at $222 \mathrm{~nm}$ and $208 \mathrm{~nm}\left([\theta]_{208} /[\theta]_{222}\right)$, which is commonly used for the quantitative analysis of the $\alpha$ helical structure, was $1.01,1.02,0.99$, and 0.98 for fibrinogen, fibrinogen $+\mathrm{COOH}-1$, fibrinogen $+\mathrm{COOH}-2$, and fibrinogen + $\mathrm{COOH}-3$ swCNT, respectively. The addition of $\mathrm{COOH}-1$ nanoparticle to fibrinogen elicited only a slight reduction in the CD signal and a slight enhancement of the intensity ratio, indicating that the $\alpha$ helical structure in the presence of $\mathrm{COOH}-1$ resembled that of free fibrinogen. However, $\mathrm{COOH}-2$ and -3 nanoparticle mixtures gave comparable changes in intensities and intensity ratio (Figure $3 \mathrm{~A}$ and $\mathrm{B}$ ). Highly dispersed states enhanced the separation of swCNTs and reduced aggregation of CNT by increasing the number of carboxyl groups presented at the CNT surface, which may have induced more structural changes of fibrinogen.

The changes of secondary structural components are presented in Figure 3B based on the nanoparticle mixture. Notably, the amount of $\alpha$ helix decreased and the amount of $\beta$ strand increased by a similar percentage in both the $\mathrm{COOH}-2$ and -3 swCNT mixtures (Figure 3B). This data supports the idea of transformation of $\alpha$ helices into $\beta$ sheet secondary structures, as hypothesized in theoretical analyses. ${ }^{20}$

Protein adsorption onto the synthetic surface was shown to depend on the hydrophobicity and electrostatic attraction at the surface. Morin et al demonstrated that fibrinogen adsorption preferentially occurred on more 
A

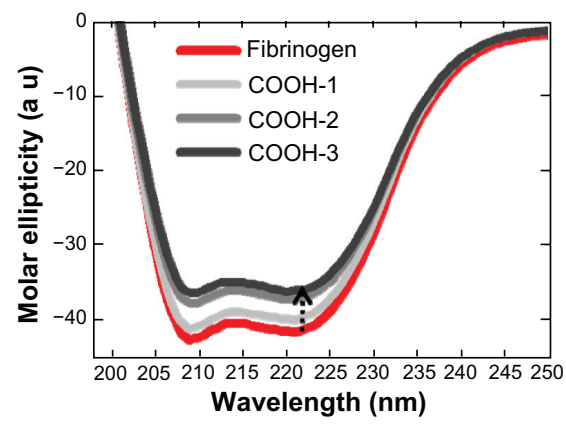

C

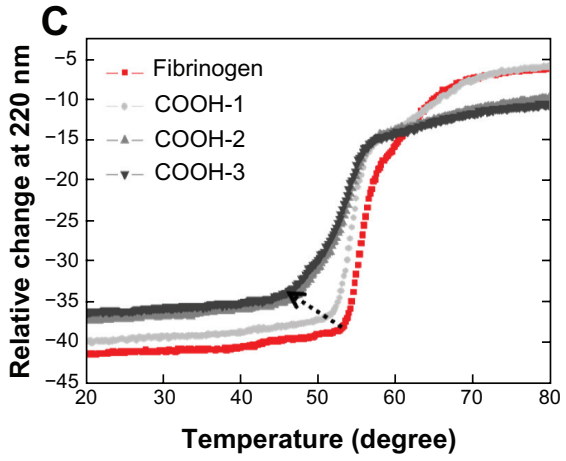

B

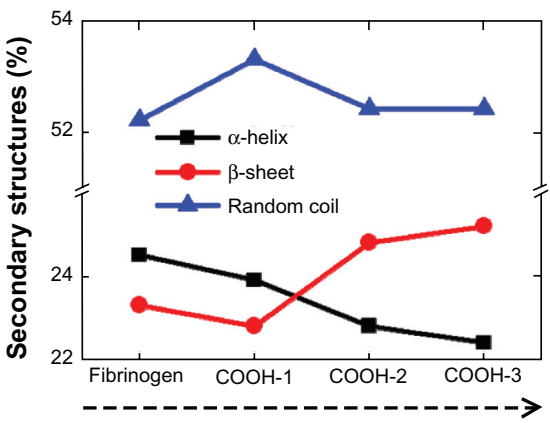

D

Higher dispersion

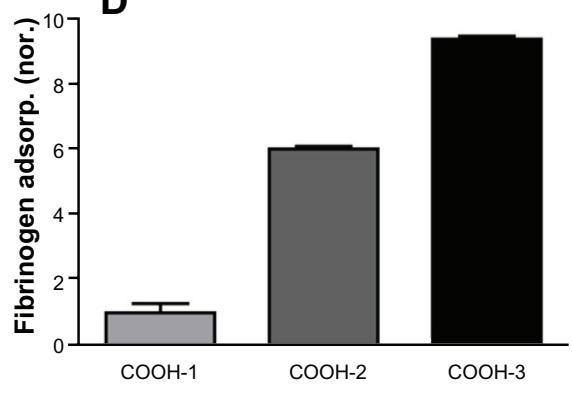

Figure 3 The conformational changes of fibrinogen in terms of the dispersion rate of swCNTs. (A) The effect of different orders of carboxyl functional groups of CNTs on the CD spectra of fibrinogen. (B) Changes of secondary structures. The amount of $\alpha$-helical content decreased while the amount of $\beta$-strand content increased in the $\mathrm{COOH}-2$ and -3 swCNT mixtures. This data represented evidence of switching from $\alpha$-helix to $\beta$-strand. (C) The impact of carboxyl order on the conformational change of fibrinogen is demonstrated on CD temperature scans. Unfolding of fibrinogen in terms of dispersion rate (relative change) shows early phase transition as dispersion rate is increased in swCNTs. (D) The adsorbed amount of fibrinogen as dispersion is increased in swCNTs. At an identical density, there is an almost ten time increment of adsorbed fibrinogen on $\mathrm{COOH}-3$ compared to $\mathrm{COOH}-\mathrm{I}$ due to increased dispersion.

Abbreviation: $\mathrm{CD}$, circular dichroism.

hydrophobic polystyrene surfaces. ${ }^{22}$ Several studies have shown that electrostatic interaction of the proteins is important when considering protein selectivity for a synthetic surface. ${ }^{23,24}$ In the present study, fibrinogen had an isoelectric point value of 5.5, demonstrating a net negative charge in the given experimental condition. Furthermore, the addition of carboxyl groups introduced a net negative charge to the swCNTs. Thus, at physiological $\mathrm{pH}$, the global interaction is predicted to be repulsive. However, our CD data demonstrated that an increase in carboxyl weight of the CNTs promoted the conformational change of fibrinogen, which emphasizes the structural effects of proteins interacting with CNTs. As shown in Figure 4, the negatively charged surfaces are concentrated on one face (Figure 4A) where the interaction is probably repulsive, while the distribution of positive charge is relatively limited on the other face where the positively charged regions appear locally along the $\alpha$ helical rod (Figure 4B). Considering hydrophobicity as well as polarity of nanotubes is important for the interaction, since the result might point towards selective localization of fibrinogen on the surface of nanotubes. In addition, there are some missing parts in the current crystal structure of fibrinogen. Those include the missing $\mathrm{C}$-terminal region of the $\mathrm{A} \alpha$ chain consisting of 362 amino acids, the missing $\mathrm{N}$-terminal region of the $\mathrm{B} \beta$ chain consisting of 57 amino acids with 7 negatively-charged residues, and 12 positively-charged residues. In particular the latter region might affect localization of fibrinogen on

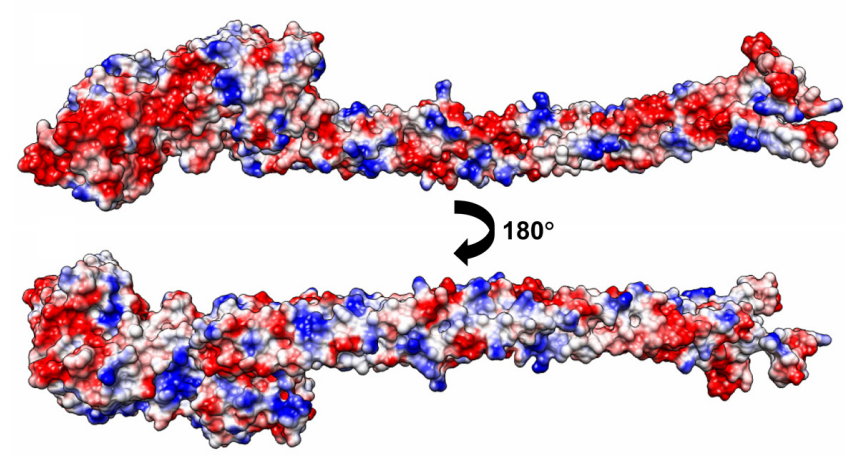

Figure 4 Electrostatic potential surface diagrams of a fibrinogen $(A \alpha B \beta \gamma)_{2}$ monomer shows a local concentration of positive charge along the coiled-coil rod facing outwards.

Note: The columbic parameters were $\varepsilon=4 \mathrm{r}$ and thresholds $\pm 5.93 \mathrm{kcal} / \mathrm{mol} \cdot \mathrm{e}$. Negative charge is indicated in red, positive blue, and neutral white. The surface charge was calculated with UCSF Chimera. ${ }^{27}$

Abbreviations: $\varepsilon$, dielectric constant; $r$, distance. 
the surface of nanotubes since the net charge is apparently positive.

In addition, no significant changes in random coils were observed when fibrinogen came in contact with the COOH-1, $\mathrm{COOH}-2$ and $\mathrm{COOH}-3$ samples (Figure 3B). This implied that no significant structural changes manifested in unordered regions of fibrinogen. Unordered regions are found in the $\mathrm{N}$-terminus of the $\alpha$ and $\beta$ chains and the $\mathrm{C}$-terminal domains of the $\beta$ and $\gamma$ chains. ${ }^{25}$ Thus, the structures of these regions might not be heavily influenced by fibrinogen interactions with swCNTs of varying dispersion, even though it is important for the localization of fibrinogen on nanotubes.

The impact of carboxyl order on the conformational change of fibrinogen is additionally demonstrated on $\mathrm{CD}$ temperature scans (Figure 3C). Free fibrinogen showed two clear conformational states during heat denaturation. Denaturation of fibrinogen begins at $53^{\circ} \mathrm{C}$ and proceeds with a steep slope until $59^{\circ} \mathrm{C}$ is reached (Figure 3C). At this temperature, the second transition may take place, the slope decreasing noticeably as the temperature rises to approximately $70^{\circ} \mathrm{C}$ when the fibrinogen is completely unfolded. The melting temperature $\left(\mathrm{T}_{\mathrm{m}}\right)$ of free fibrinogen is approximately $61.5^{\circ} \mathrm{C}$. Previous studies have revealed two denaturation transitions of native fibrinogen at $61^{\circ} \mathrm{C}$ and $100^{\circ} \mathrm{C} .{ }^{26}$ The pattern of denaturation observed in mixtures of fibrinogen and $\mathrm{COOH}-1$ resembled that of native fibrinogen, but with a slightly lower melting temperature consistent with the $\mathrm{CD}$ temperature scans. As expected, fibrinogen mixtures with $\mathrm{COOH}-2$ and -3 swCNTs demonstrated comparably lower $\mathrm{T}_{\mathrm{m}}$ values; the denaturation of fibrinogen beginning at approximately $46^{\circ} \mathrm{C}$ and lasting to $57^{\circ} \mathrm{C}$ for both mixtures. In addition, the denaturation process distinctively showed two transition states. The first transition occurred between $46^{\circ} \mathrm{C}$ and $52^{\circ} \mathrm{C}$ with a gentle slope, and the second transition occurred between $52^{\circ} \mathrm{C}$ and $57^{\circ} \mathrm{C}$ with a steep slope. In fact, a subtle transition was apparent from about $60^{\circ} \mathrm{C}$ to $70^{\circ} \mathrm{C}$, which overlapped into the final state of denaturation. This overlapping transition may be regarded as the final change of the most compact region before complete unfolding. The $\mathrm{T}_{\mathrm{m}}$ values of $\mathrm{COOH}-2$ and -3 mixtures were $51.5^{\circ} \mathrm{C}$ and $51^{\circ} \mathrm{C}$, respectively. Although all samples demonstrated two (or three) transition states, the patterns of transition exhibited unique features that distinguished free fibrinogen from mixtures with $\mathrm{COOH}-1,-2$, and -3 . These results suggest that the folding state and secondary (including higher order) structures of fibrinogen were affected by the CNTs, and that the unfolding kinetics of fibrinogen changed in response to the CNTs. For a fixed swCNT density, increasing the order of the functional groups (ie, $\mathrm{COOH}-1,-2$, and -3 ) increasingly altered the denaturation, and hence, the unfolding rate of fibrinogen.

\section{Increased adsorption of fibrinogen with increased dispersion of swCNTs}

Increasing the order of carboxyl groups corresponded to increased fibrinogen adsorption; the $\mathrm{COOH}-3$ samples demonstrating the highest adsorption of fibrinogen compared with $\mathrm{COOH}-1$ and $\mathrm{COOH}-2$ swCNTs (Figure 3D). This may be attributed to the increased effective surface area of the swCNTs (ie, less aggregated and thus, higher curvature), which promoted greater interaction with fibrinogen. In turn, more aggregated CNTs (ie, less curvature) showed a lower binding affinity with fibrinogen at identical densities of swCNTs $(50 \mu \mathrm{g} / \mathrm{mL})$. This suggested that the enhanced dispersion of swCNTs enhanced interactions with fibrinogen, and in this manner, induced greater changes in the secondary structures of fibrinogen.

\section{Conformational changes of fibrinogen with increasing density of swCNTs}

The conformational changes of fibrinogen also depended on the density of swCNTs in the sample, as observed by $\mathrm{CD}$ spectrometry (Figure $5 \mathrm{~A}$ and $\mathrm{B}$ ). The samples of COOH-3 swCNTs were prepared at varying densities (ie, 26,64 and $128 \mu \mathrm{g} / \mathrm{mL}$ ) to examine the effects of density on the interaction with fibrinogen $(750 \mu \mathrm{g} / \mathrm{mL})$. Varying the density of identically functionalized swCNTs (ie, $\mathrm{COOH}-3$ ) altered the $\mathrm{CD}$ signal of fibrinogen (Figure 5A), while addition of the COOH-3 swCNTs in fibrinogen reduced the absolute value of CD intensities at $208 \mathrm{~nm}$ and $222 \mathrm{~nm}$ without shifting their corresponding negative minima (Figure 5A). This result indicates that the density of CNT is another factor that contributes to the secondary structures of interacting fibrinogen. The ratio of CD intensities at $222 \mathrm{~nm}$ and $208 \mathrm{~nm}$ $\left([\theta]_{208} /[\theta]_{222}\right)$ decreased from 1.01 to 0.95 with increasing concentrations of swCNTs. The amount of the $\alpha$ helical content decreased, indicating that density of the swCNTs highly influenced the helical structures of fibrinogen (Figure 5B). In contrast, the amount of $\beta$ strand linearly increased and the amount of random coil slightly increased with increasing concentrations of COOH-3 swCNT (Figure 5B).

As observed in the previous section (by variation of dispersion of swCNTs), the amount of change for $\alpha$ helix and $\beta$ sheets was almost identical (Figure 5B). Consistent with previous results, density of the swCNTs demonstrated a linear effect on the unordered regions of fibrinogen. This may be attributed 



Figure 5 Conformational changes of fibrinogen in terms of concentration of swCNTs. (A) The effect of concentration of carboxylate swCNTs on CD spectra of fibrinogen. (B) Changes of secondary structures. The amount of $\alpha$-helical content decreased while the amount of $\beta$-strand content increased as the density of $\mathrm{COOH}-3$ swCNTs increased. This data represents evidence of transforming secondary structures following a similar trend in Figure 4C. The impact of carboxyl order on the conformational changes of fibrinogen is demonstrated on CD temperature scans. Unfolding of fibrinogen in terms of concentration of swCNTs shows early phase transition as swCNT concentration is increased. (D) Adsorbed amount of fibrinogen as swCNT concentration is increased. In an identical dispersion (COOH-3), the adsorption amount shows drastic increment (about 40 times) of adsorbed fibrinogen by increasing 5 times the concentration of COOH-3 swCNT.

Abbreviation: $C D$, circular dichroism.

to the increased adsorption of fibrinogen on high density COOH-3 swCNT. The dependence of conformational change on density was highlighted by temperature scan experiments (Figure 5C). As previously described, free fibrinogen distinctively denatures through at least two intermediate states. This pattern of denaturation was maintained until the concentration of COOH-3 swCNT reached $26 \mu \mathrm{g} / \mathrm{mL}$, although the $\mathrm{T}_{\mathrm{m}}$ value decreased from $61.5^{\circ} \mathrm{C}$ to $60.5^{\circ} \mathrm{C}$ (Figure 5C). Changes in the denaturation process occurred near the concentration of $64 \mu \mathrm{g} / \mathrm{mL}$, as the second state of denaturation (typically observed in free fibrinogen) was undistinguishable in the $T_{m}$ curve at this concentration of $\mathrm{COOH}-3$. Significant changes to the unfolding pattern of fibrinogen can be observed for COOH-3 concentrations exceeding $100 \mu \mathrm{g} / \mathrm{mL}$. This result emphasizes the importance of CNT density on the conformational change of interacting protein structures. In addition, the obtained results showed an identical trend with previous studies, which reported that increasing the density of functionalized swCNTs can elicit conformational changes in the secondary structure of albumin. ${ }^{21}$ Furthermore, the observed trend of this study (relationship between CNT density and fibrinogen structure) aligns with the previous findings based on gold nanoparticles. ${ }^{7}$

\section{Increased adsorption of fibrinogen by increasing the density of swCNTs}

The adsorption of fibrinogen on swCNTs increased with increasing density of COOH-3 swCNTs (Figure 5D). This was attributed to the enlarged effective surface area achieved by increasing the amount of CNTs in a given volume; the increase in surface area promoting greater interactions with fibrinogen at higher concentrations (eg, $128 \mu \mathrm{g} / \mathrm{mL}$ ). CNT samples had identical curvature (or same dimensions both in length and radius) but at lower concentrations (eg, 26 and $64 \mu \mathrm{g} / \mathrm{mL}$ ) corresponding to a lower binding affinity with fibrinogen $(1 \mathrm{mg} / \mathrm{mL})$ than samples at higher concentrations of COOH-3 (eg, $128 \mu \mathrm{g} / \mathrm{mL})$.

\section{Conclusion}

In this study, we identified that the concentration and dispersion of swCNTs were significant material surface properties with respect to the conformational state and the structural stability of fibrinogen. Although the size (ie, curvature) of the carboxylate carbon nanotubes is linked with surface chemistry, the obtained results demonstrate that the dispersion of swCNTs, as determined by aggregation and concentration of the carbon nanotubes, influences the conformational change 
of secondary fibrinogen structures as well as the unfolding process. Higher density and dispersion of CNT samples corresponded to lower melting temperatures, whereas low density and less dispersed CNT exhibited higher melting temperatures that approximated the melting point of free fibrinogen. In conclusion, the understanding and control of physiochemical surface properties of nanomaterials can control the conformational changes of contacted proteins and influence the denaturation of plasma proteins, ultimately dictating cell fate and functions.

\section{Acknowledgments}

Thanks to Melisa Tsang (Biomedical Engineering, Georgia Institute of Technology) for editing. This research was supported by PRC (2012-0001054) and the basic science research program through the National Research Foundation of Korea (NRF) funded by the Ministry of Education, Science and Technology (2011-0011603, 2011-0002403 and 2011-0006036).

\section{Disclosure}

The authors state that there is no conflict of interest in the work.

\section{References}

1. Chen RJ, Bangsaruntip S, Drouvalakis KA, et al. Noncovalent functionalization of carbon nanotubes for highly specific electronic biosensors. Proc Natl Acad Sci U S A. 2003;100(9):4984-4989.

2. Deng ZJ, Liang M, Monteiro M, Toth I, Minchin RF. Nanoparticleinduced unfolding of fibrinogen promotes Mac-1 receptor activation and inflammation. Nat Nanotechnol. 2011;6(1):39-44.

3. Lundqvist M, Stigler J, Elia G, et al. Nanoparticle size and surface properties determine the protein corona with possible implications for biological impacts. Proc Natl Acad Sci U SA. 2008;105(38):14265-14270.

4. Stayton PS, Hoffman AS, Murthy N, et al. Molecular engineering of proteins and polymers for targeting and intracellular delivery of therapeutics. J Control Release. 2000;65(1-2):203-220.

5. Peer D, Karp JM, Hong S, et al. Nanocarriers as an emerging platform for cancer therapy. Nat Nanotechnol. 2007;2(12):751-760.

6. Liu Z, Robinson JT, Tabakman SM, Yang K, Dai H. Carbon materials for drug delivery and cancer therapy. Mater Today. 2011;14(7-8): 316-323.

7. Shao Q, Wu P, Gu P, Xu X, Zhang H, Cai C. Electrochemical and spectroscopic studies on the conformational structure of hemoglobin assembled on gold nanoparticles. J Phys Chem B. 2011;115(26): 8627-8637.
8. Ge C, Du J, Zhao L, et al. Binding of blood proteins to carbon nanotubes reduces cytotoxicity. Proc Natl Acad Sci U S A. 2011;108(41): 16968-16973.

9. Dobrovolskaia MA, Germolec DR, Weaver JL. Evaluation of nanoparticle immunotoxicity. Nat Nanotechnol. 2009;4(7):411-414.

10. Nel AE, Madler L, Velegol D, et al. Understanding biophysicochemical interactions at the nano-bio interface. Nat Mater. 2009;8(7): 543-557.

11. Xia XR, Monteiro-Riviere NA, Riviere JE. An index for characterization of nanomaterials in biological systems. Nat Nanotechnol. 2010;5(9): 671-675.

12. Lynch I, Dawson KA. Protein-nanoparticleinteractions. Nano Today. 2008;3(1-2):40-47.

13. Flick M, Du X, Degen J. Fibrin(ogen)-alpha M beta 2 interactions regulate leukocyte function and innate immunity in vivo. Exp Biol Med (Maywood). 2004;229(11):1105-1110.

14. Weisel JW. Fibrinogen and fibrin. Adv Protein Chem. 2005;70:247-299.

15. Soderqvist T, Blomback B. Fibrinogen structure and evolution. Naturwissenschaften. 1971;58(1):16-23.

16. Doolittle RF. Fibrinogen and fibrin. Annu Rev Biochem. 1984;53: 195-229.

17. Roach P, Farrar D, Perry CC. Surface tailoring for controlled protein adsorption: effect of topography at the nanometer scale and chemistry. J Am Chem Soc. 2006;128(12):3939-3945.

18. Shen JW, Wu T, Wang Q, Kang Y. Induced stepwise conformational change of human serum albumin on carbon nanotube surfaces. Biomaterials. 2008;29(28):3847-3855.

19. Balamurugan K, Gopalakrishnan R, Raman SS, Subramanian V. Exploring the changes in the structure of alpha-helical peptides adsorbed onto a single walled carbon nanotube using classical molecular dynamics simulation. J Phys Chem B. 2010;114(44):14048-14058.

20. Ou L, Luo Y, Wei G. Atomic-level study of adsorption, conformational change, and dimerization of an alpha-helical peptide at graphene surface. J Phys Chem B. 2011;115(32):9813-9822.

21. Zhao X, Liu R, Chi Z, Teng Y, Qin P. New insights into the behavior of bovine serum albumin adsorbed onto carbon nanotubes: comprehensive spectroscopic studies. J Phys Chem B. 2010;114(16):5625-5631.

22. Morin C, Hitchcock AP, Cornelius R, et al. Selective adsorption of protein on polymer surfaces studied by soft X-ray photoemission electron microscopy. J Electron Spectroscopy. 2004;137-140:785-794.

23. Kondo A, Higashitani K. Adsorption of model proteins with wide variation in molecular properties on colloidal particles. $J$ Colloid Interface Sci. 1992;150(2):344-351.

24. Nakanishi K, Sakiyama T, Imamura K. On the adsorption of proteins on solid surfaces, a common but very complicated phenomenon. $J$ Biosci Bioeng. 2001;91(3):233-244.

25. Kollman JM, Pandi L, Sawaya MR, Riley M, Doolittle RF. Crystal structure of human fibrinogen. Biochemistry. 2009;48(18):3877-3886

26. Donovan JW, Mihalyi E. Conformation of fibrinogen: calorimetric evidence for a three-nodular structure. Proc Natl Acad Sci U SA. 1974; 71(10):4125-4128.

27. Pettersen EF, Goddard TD, Huang CC, et al. UCSF Chimera a visualization system for exploratory research and analysis. $J$ Comput Chem. 2004;25(13):1605-1612.
International Journal of Nanomedicine

\section{Publish your work in this journal}

The International Journal of Nanomedicine is an international, peerreviewed journal focusing on the application of nanotechnology in diagnostics, therapeutics, and drug delivery systems throughou the biomedical field. This journal is indexed on PubMed Central, MedLine, CAS, SciSearch ${ }^{\circledR}$, Current Contents ${ }^{\circledR} /$ Clinical Medicine,

\section{Dovepress}

Journal Citation Reports/Science Edition, EMBase, Scopus and the Elsevier Bibliographic databases. The manuscript management system is completely online and includes a very quick and fair peer-review system, which is all easy to use. Visit http://www.dovepress.com/ testimonials.php to read real quotes from published authors. 